\title{
FRANJAS MARGINALES DE Brassica campestris L. (NABO) EN CULTIVO DE REPOLLO. EFECTO SOBRE PULGONES Y SUS PARASITOIDES
}

\author{
Curis, M. C. ${ }^{1 ;}$ Saravia steudtner, F. ${ }^{1 ;}$ FaVaro, J. C. ${ }^{1}$; \\ SÁNCHEZ, D. ${ }^{1} \&$ BERTOLACCINI, I. ${ }^{1}$
}

\begin{abstract}
RESUMEN
La diversidad vegetal en los agroecosistemas afecta la dinámica poblacional de las plagas y de sus enemigos naturales, siendo una alternativa de control el uso de franjas trampa en algunos cultivos. El objetivo del trabajo fue determinar como una franja marginal de Brassicas campestris L. (Brassicales, Brassicaceae), afecta a la población de los pulgones de B. oleracea var. capitata y de sus parasitoides. El estudio se llevó a cabo en Santa Fe, a partir de febrero de 2012. Se estableció en un cultivo comercial de repollo, y en el lado norte del mismo una franja marginal con colza. Se realizaron cuatro tratamientos: 1) con franja marginal, sin usote agroquímicos; 2) con franja marginal, con agroquímicos; 3) sin franja marginal, sin agroquímicos, 4) sin franja marginal, con agroquímicos. Semanalmente se monitoreó la presencia de áfidos y de parasitoides en tres plantas por tratamiento hallándose diferencias significativas en las poblaciones en las franjas marginales y el cultivo.

Palabras clave: Aphididae, biodiversidad, control biológico, Brevicoryne brassicae, Mizus persicae, Diaeretiella rapae.
\end{abstract}

\section{SUMMARY}

\section{Marginal stripes of Brassica campestris L. in cabbage crop: aphids and} parasitoids. Effects.

The dynamics of population of pests and natural enemies are affected by plant diversity in agroecosystems; being an alternative the use strips traps to control in several crops. The aim of this work was to determine the effect of marginal strips of Brassica campestris on the aphid population and their parasitoids in a cabbage (Brassica oleracea var. capitata) crop. The study was conducted in Santa Fe area, in a commercial crop, beginning on February 2012. At the northern edge, the strip

1.- Departamento de Producción Vegetal, Facultad de Ciencias Agrarias (UNL). Kreder 2805. (3080) Esperanza, provincia de Santa Fe. Email: isabelb@fca.unl.edu.ar. Organismo otorgante: Universidad Nacional del Litoral, a través de los Proyectos CAI+D 2009.

Manuscrito recibido el 19 de septiembre de 2013 y aceptado para su publicación el 2 de agosto de 2014. 


\section{C. Curis et al.}

trap was made with plants of rape oil. Four treatments were made: 1) marginal strip, without use of agrochemicals, 2) marginal strip, with agrochemicals, 3) no marginal strip without agrochemicals and 4) no marginal strip, with agrochemical. Weekly was monitored the presence of aphids and parasitoids, in three plant per treatment, finding significant differences in the populations present in the crop and marginal strip. Weekly was monitored the presence of aphids and parasitoids, in three plant per treatment, finding significant differences in the populations present in the crop and marginal strip.

Key words: Aphididae, biodiversity, control biológico, Brevicoryne brassicae, Mizus persicae, Diaeretiella rapae.

\section{INTRODUCCIÓN}

El repollo (Brassica oleracea var. capitata) es un cultivo hortícola de gran importancia a nivel mundial. Los pulgones pertenecientes a la superfamilia Aphidoidea se consideran como una de las plagas más perjudiciales y de aparición constante en cultivos de la familia Brassicaceae (1). Las especies más importantes a nivel mundial pertenecen a la familia Aphididae son Brevicoryne brassicae L., Lipaphis erysimi (Kalt.) y Myzus persicae (Sulzer), los cuales, además de los daños directos que causan por su alimentación (extracción de savia e inyección de salivas tóxicas), son vectores de virosis $(2,3)$. Generalmente sus poblaciones son controladas con insecticidas de síntesis, cuya utilización intensiva provoca, contaminación del medio, resistencia en las plagas, entre otros, razón por la cual en los últimos años, los estudios tienden al desarrollo de técnicas que favorezcan una producción sustentable, con un menor uso de plaguicidas (4).

En nuestro país uno de los problemas de mayor incidencia en los cultivos de brasicas es la presencia de $B$. brassicae al que se considera autoico por la alta especificidad con su huésped $(5,6)$, mientras que Myzus persicae es cosmopolita adaptado a un amplio rango de hospederos, tanto cultivados como silvestres $(7,8)$. De estos pulgones $B$. brassicae posee buenos parámetros reproductivos en el repollo mientras que $M$. persicae, a pesar de ser polífago, demostró tener un comportamiento reproductivo similar al pulgón de las crucíferas dependiendo del hospedero (9).

Los áfidos cuentan con enemigos naturales, entre los que se encuentran predadores, como los coccinélidos y los sírfidos e himenópteros parasitoides (10); dentro de estos últimos la especie Diaeretiella rapae M'Intosh (Hymenoptera: Braconidae) es de frecuente aparición atacando a las especies que se alimentan de repollo (11).

El control que ejercen los parasitoides ha sido muy estudiado (12). La hembra utiliza su ovipositor para colocar un huevo dentro del pulgón, de éste emerge una larva la cual se va alimentando de su hospedador a medida que van pasando los distintos estadios de su desarrollo. El pulgón se hincha y va perdiendo movilidad hasta quedar completamente rígido y fijado a la planta, en esta etapa recibe el nombre de "momia". Finalmente de la momia mediante un orificio autoinducido, emerge el adulto del parasitoide $(12,13,14)$. En 